Document downloaded from:

http://hdl.handle.net/10251/155704

This paper must be cited as:

Chicharro López, FI.; Cordero Barbero, A.; Torregrosa Sánchez, JR. (2019). Dynamics of iterative families with memory based on weight functions procedure. Journal of Computational and Applied Mathematics. 354:286-298.

https://doi.org/10.1016/j.cam.2018.01.019

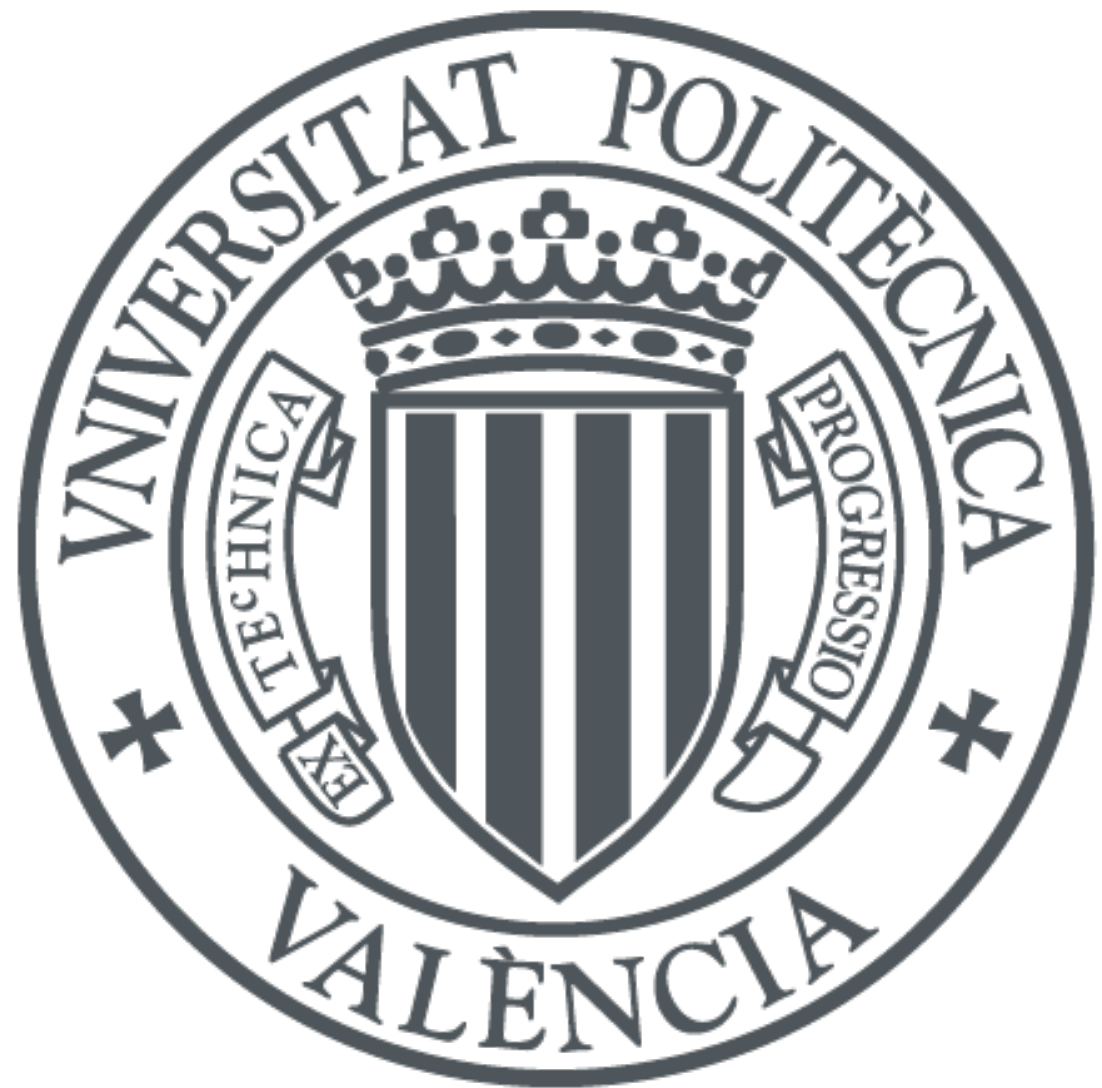

The final publication is available at

https:/doi.org/10.1016/j.cam.2018.01.019

Copyright Elsevier

Additional Information 


\title{
Dynamics of iterative families with memory based on weight functions procedure $^{\text {市 }}$
}

\author{
Francisco I. Chicharro ${ }^{\mathrm{a}}$, Alicia Cordero ${ }^{\mathrm{a}}$, Juan R. Torregrosa ${ }^{\mathrm{a}}$ \\ ${ }^{a}$ Instituto Universitario de Matemática Multidisciplinar \\ Universitat Politècnica de València, \\ Camino de Vera s/n, 46022 València, Spain
}

\begin{abstract}
In this paper, we analyze the stability of a parametric family of iterative methods with memory for solving nonlinear equations. This family is obtained from an optimal class of fourth-order schemes without memory designed by means of weight functions procedure. By studying the real fixed and critical points of the rational function resulting from the application of the family with memory on quadratic polynomials, the best elements of the family, in terms of absence of chaotic behavior, are selected. Finally, a numerical study is performed verifying the dynamical theoretical results.
\end{abstract}

Keywords: Nonlinear equations, acceleration of convergence, methods with memory, real dynamics, bifurcation diagram.

2010 MSC: 34A34, 65B99, 65P30, 65H05.

\section{Introduction}

Nonlinearity is ubiquitous in physical phenomena as fluid and plasma mechanics, gas dynamics, elasticity, relativity, chemical reactions, combustion, ecology, biomechanics, economics modeling problems, transport theory and many other problems that are modeled by nonlinear equations. So, the design of fixed point iterative methods for solving nonlinear equations $f(x)=0$ is a challenging task in Numerical Analysis.

The iterative methods with memory use information from the current iteration and the previous ones. The key point of this kind of methods is the increasing of the order of convergence of the original scheme without adding new functional evaluations. This is often based on the existence of accelerating parameters that are involved in the error equation of the original methods.

The first method with memory including accelerators is based on Steffensen's method [1] and was designed by Traub in 2. He developed a method with memory with slight but significant changes of Steffensen's method, resulting in an order of convergence of 2.41 .

Despite there are methods with memory that include expressions with derivatives (see, for example [3, 4, 5]), the most common practice is to design derivative-free iterative methods with memory. There is an extensive literature of this sort of schemes, standing out the papers of Petković and Džunic $(\underline{6}, 7,8,9])$, or by other authors such as Lotfi, Soleymani, Sharma or Zafar ([10, 11, 12, 13, 14, 15]) by using similar techniques.

The main goal of this study is the in-depth analysis of the real dynamics of a new iterative family of methods with memory designed in this work.

约 This research was partially supported by both Ministerio de Economía y Competitividad and Generalitat Valenciana, under grants MTM2014-52016-C2-2-P and PROMETEO/2016/089, respectively.

Email addresses: frachilo@upvnet.upv.es (Francisco I. Chicharro), acordero@mat.upv.es (Alicia Cordero), jrtorre@mat.upv.es (Juan R. Torregrosa) 
Taking King's family of optimal fourth-order iterative methods as a starting point,

$$
\begin{aligned}
& y_{k}=x_{k}-\frac{f\left(x_{k}\right)}{f^{\prime}\left(x_{k}\right)}, \\
& x_{k+1}=y_{k}-\frac{f\left(x_{k}\right)+\alpha f\left(y_{k}\right)}{f\left(x_{k}\right)+(\alpha-2) f\left(y_{k}\right)} \frac{f\left(y_{k}\right)}{f^{\prime}\left(x_{k}\right)},
\end{aligned}
$$

where $\alpha$ is a free real parameter, we introduce a family of derivative-free methods using the weight function technique. The error equation of this class allows us to construct a family with memory by estimating the accelerated parameter, in each iteration, by means of available information. In order to analyze its convergence we use the following result [16.

Theorem 1. Let $\psi$ be an iterative method with memory that generates a sequence $\left\{x_{k}\right\}$ of approximations to the root $x^{*}$, and let this sequence converge to $x^{*}$. If there exists a nonzero constant $K$ and nonnegative numbers $r_{i}$, $i=0,1, \ldots, R$, such that the inequality

$$
\left|e_{k+1}\right| \leq K \prod_{i=0}^{R}\left|e_{k-i}\right|^{r_{i}}
$$

holds, then the R-order of convergence of the iterative method $\psi$ satisfies the inequality

$$
O_{R}\left(\psi, x^{*}\right) \geq v^{*}
$$

where $v^{*}$ is the unique positive root of the equation

$$
v^{R+1}-\sum_{i=0}^{R} r_{i} v^{R-i}=0 .
$$

The paper is organized as follows. In Section 2, a family of iterative methods without memory is introduced and its fourth-order convergence is proven. Moreover, some particular cases are stated. Section 3 is devoted to the design of a family of iterative methods with memory starting from the previous without memory partner. In Section 4 a real dynamical analysis is performed to check the behavior of these schemes with memory and select those with better stability properties. Finally, Section 5 covers the numerical experience on different tests functions.

\section{Families of iterative methods without memory}

From King's family (1), replacing the derivative by different first-order divided differences, and considering the first factor of the second step as a weight function $H(t)$, we introduce the schemes

$$
\begin{aligned}
y_{k} & =x_{k}-\frac{f\left(x_{k}\right)}{f\left[x_{k}, w_{k}\right]}, \\
x_{k+1} & =y_{k}-H\left(t_{k}\right) \frac{f\left(y_{k}\right)}{f\left[y_{k}, w_{k}\right]},
\end{aligned}
$$

where $H(t)$ is the weight function of variable $t=f(y) / f(x)$ and $w=x+\gamma f(x)$, being $\gamma$ a nonzero parameter. Its order of convergence is established in the following result.

Theorem 2. Let us suppose that $f: I \subset \mathbb{R} \rightarrow \mathbb{R}$ is a sufficiently differentiable function in an open interval $I$ and $x^{*} \in I$ is a simple root of $f(x)=0$. If the initial approximation $x_{0}$ is close enough to $x^{*}$ and function $H(t)$ satisfies the conditions $H(0)=H^{\prime}(0)=1$ and $H^{\prime \prime}(0)<\infty$, then the iterative schemes in (2) have optimal fourth-order of convergence, for all nonzero $\gamma$, being in this case the error equation

$$
e_{k+1}=-\frac{c_{2}}{2}\left(1+\gamma f^{\prime}\left(x^{*}\right)\right)^{2}\left[\left(-6+\gamma f^{\prime}\left(x^{*}\right)\left(H^{\prime \prime}(0)-2\right)+H^{\prime \prime}(0)\right) c_{2}^{2}+2 c_{3}\right] e_{k}^{4}+\mathcal{O}\left(e_{k}^{5}\right),
$$

where $e_{k}=x_{k}-x^{*}, k=0,1, \ldots$ and $c_{j}=\frac{1}{j !} \frac{f^{(j)}\left(x^{*}\right)}{f^{\prime}\left(x^{*}\right)}$. 
Proof. By expanding $f\left(x_{k}\right)$ in Taylor series,

$$
f\left(x_{k}\right)=f^{\prime}\left(x^{*}\right)\left[e_{k}+c_{2} e_{k}^{2}+c_{3} e_{k}^{3}+c_{4} e_{k}^{4}\right]+\mathcal{O}\left(e_{k}^{5}\right) .
$$

The expansion of the divided difference $f\left[x_{k}, w_{k}\right]$ is

$$
\begin{aligned}
f\left[x_{k}, w_{k}\right]= & f^{\prime}\left(x^{*}\right)\left[1+\left(2+\gamma f^{\prime}\left(x^{*}\right)\right) c_{2} e_{k}+\left(\gamma c_{2}^{2} f^{\prime}\left(x^{*}\right)+c_{3}\left(3+3 \gamma f^{\prime}\left(x^{*}\right)+\gamma^{2} f^{\prime}\left(x^{*}\right)^{2}\right)\right) e_{k}^{2}\right. \\
& \left.+\left(2+\gamma f^{\prime}\left(x^{*}\right)\right)\left(2 c_{2} c_{3} \gamma f^{\prime}\left(x^{*}\right)+c_{4}\left(2+2 \gamma f^{\prime}\left(x^{*}\right)+\gamma^{2} f^{\prime}\left(x^{*}\right)^{2}\right)\right) e_{k}^{3}\right]+\mathcal{O}\left(e_{k}^{4}\right),
\end{aligned}
$$

and the error in the first step yields

$$
\begin{aligned}
y_{k}-x^{*}= & c_{2}\left(1+\gamma f^{\prime}\left(x^{*}\right)\right) e_{k}^{2}+\left[-c_{2}^{2}\left(2+2 \gamma f^{\prime}\left(x^{*}\right)+\gamma^{2} f^{\prime}\left(x^{*}\right)^{2}\right)+c_{3}\left(2+3 \gamma f^{\prime}\left(x^{*}\right)+\gamma^{2} f^{\prime}\left(x^{*}\right)^{2}\right)\right] e_{k}^{3} \\
& +\left[c_{2}^{3}\left(4+5 \gamma f^{\prime}\left(x^{*}\right)+3 \gamma^{2} f^{\prime}\left(x^{*}\right)^{2}+\gamma^{3} f^{\prime}\left(x^{*}\right)^{3}\right)-c_{2} c_{3}\left(7+10 \gamma f^{\prime}\left(x^{*}\right)+7 \gamma^{2} f^{\prime}\left(x^{*}\right)^{2}+2 \gamma^{3} f^{\prime}\left(x^{*}\right)^{3}\right)\right. \\
& \left.\left.+c_{4}\left(3+6 \gamma f^{\prime}\left(x^{*}\right)+\right)+4 \gamma^{2} f^{\prime}\left(x^{*}\right)^{2}+\gamma^{3} f^{\prime}\left(x^{*}\right)^{3}\right)\right] e_{k}^{4}+\mathcal{O}\left(e_{k}^{5}\right) .
\end{aligned}
$$

The Taylor expansion of $f\left(y_{k}\right)$ results in

$$
\begin{aligned}
f\left(y_{k}\right)= & f^{\prime}\left(x^{*}\right)\left[c_{2}\left(1+\gamma f^{\prime}\left(x^{*}\right)\right) e_{k}^{2}+\left(-c_{2}^{2}\left(2+2 \gamma f^{\prime}\left(x^{*}\right)+\gamma^{2} f^{\prime}\left(x^{*}\right)^{2}\right)+c_{3}\left(2+3 \gamma f^{\prime}\left(x^{*}\right)+\gamma^{2} f^{\prime}\left(x^{*}\right)^{2}\right)\right) e_{k}^{3}\right. \\
& +\left(c_{2}^{3}\left(5+7 \gamma f^{\prime}\left(x^{*}\right)+4 \gamma^{2} f^{\prime}\left(x^{*}\right)^{2}+\gamma^{3} f^{\prime}\left(x^{*}\right)^{3}\right)-c_{2} c_{3}\left(7+10 \gamma f^{\prime}\left(x^{*}\right)+7 \gamma^{2} f^{\prime}\left(x^{*}\right)^{2}+2 \gamma^{3} f^{\prime}\left(x^{*}\right)^{3}\right)\right) \\
& \left.\left.+c_{4}\left(3+6 \gamma f^{\prime}\left(x^{*}\right)+4 \gamma^{2} f^{\prime}\left(x^{*}\right)^{2}+\gamma^{3} f^{\prime}\left(x^{*}\right)^{3}\right)\right) e_{k}^{4}\right]+\mathcal{O}\left(e_{k}^{5}\right) .
\end{aligned}
$$

Assuming that $x_{k}$ is sufficiently close to the zero $x^{*}$ of $f$, then $t_{k}$ is close enough to 0 , so we expand $H(t)$ about 0 ,

$$
H(t) \approx H(0)+H^{\prime}(0) t+\frac{1}{2} H^{\prime \prime}(0) t^{2} .
$$

By direct division, we get

$$
\begin{aligned}
\frac{f\left(y_{k}\right)}{f\left(x_{k}\right)}= & c_{2}\left(1+\gamma f^{\prime}\left(x^{*}\right)\right) e_{k}+\left[-c_{2}^{2}\left(3+3 \gamma f^{\prime}\left(x^{*}\right)+\gamma^{2} f^{\prime}\left(x^{*}\right)^{2}\right)+\left(2+3 \gamma f^{\prime}\left(x^{*}\right)+\gamma^{2} f^{\prime}\left(x^{*}\right)^{2}\right)\right] e_{k}^{2} \\
& +\left[c_{2}^{3}\left(8+10 \gamma f^{\prime}\left(x^{*}\right)+5 \gamma^{2} f^{\prime}\left(x^{*}\right)^{2}+\gamma^{3} f^{\prime}\left(x^{*}\right)^{3}\right)-2 c_{2} c_{3}\left(5+7 \gamma f^{\prime}\left(x^{*}\right)+4 \gamma^{2} f^{\prime}\left(x^{*}\right)^{2}+\gamma^{3} f^{\prime}\left(x^{*}\right)^{3}\right)\right. \\
& \left.+c_{4}\left(3+6 \gamma f^{\prime}\left(x^{*}\right)+4 \gamma^{2} f^{\prime}\left(x^{*}\right)^{2}+\gamma^{3} f^{\prime}\left(x^{*}\right)^{3}\right)\right] e_{k}^{3}+\mathcal{O}\left(e_{k}^{4}\right),
\end{aligned}
$$

so $H(t)$ yields

$$
\begin{aligned}
H(t)= & H(0)+c_{2} H^{\prime}(0)\left(1+\gamma f^{\prime}\left(x^{*}\right)\right) e_{k} \\
& +\left[\frac{c_{2}^{2}}{2}\left(H^{\prime \prime}(0)\left(1+\gamma f^{\prime}\left(x^{*}\right)\right)^{2}-2 H^{\prime}(0)\left(3+3 \gamma f^{\prime}\left(x^{*}\right)+\gamma^{2} f^{\prime}\left(x^{*}\right)^{2}\right)\right)+c_{3} H^{\prime}(0)\left(2+3 \gamma f^{\prime}\left(x^{*}\right)+\gamma^{2} f^{\prime}\left(x^{*}\right)^{2}\right)\right] e_{k}^{2} \\
& +\left[c_{2}^{3}\left(H^{\prime \prime}(0)\left(3+6 \gamma f^{\prime}\left(x^{*}\right)+4 \gamma^{2} f^{\prime}\left(x^{*}\right)^{2}+\gamma^{3} f^{\prime}\left(x^{*}\right)^{3}\right)+H^{\prime}(0)\left(8+10 \gamma f^{\prime}\left(x^{*}\right)+5 \gamma^{2} f^{\prime}\left(x^{*}\right)^{2}+\gamma^{3} f^{\prime}\left(x^{*}\right)^{3}\right)\right)\right. \\
& +c_{2} c_{3}\left(H^{\prime \prime}(0)\left(1+\gamma f^{\prime}\left(x^{*}\right)\right)^{2}\left(2+\gamma f^{\prime}\left(x^{*}\right)\right)+2 H^{\prime}(0)\left(5+7 \gamma f^{\prime}\left(x^{*}\right)+4 \gamma^{2} f^{\prime}\left(x^{*}\right)^{2}+\gamma^{3} f^{\prime}\left(x^{*}\right)^{3}\right)\right) \\
& \left.+c_{4} H(0)\left(3+6 \gamma f^{\prime}\left(x^{*}\right)+4 \gamma^{2} f^{\prime}\left(x^{*}\right)^{2}+\gamma^{3} f^{\prime}\left(x^{*}\right)^{3}\right)\right] e_{k}^{3}+\mathcal{O}\left(e_{k}^{4}\right) .
\end{aligned}
$$

A previous step for obtaining the error equation, is the calculation of $f\left[y_{k}, w_{k}\right]$ as follows

$$
\begin{aligned}
f\left[y_{k}, w_{k}\right]= & f^{\prime}\left(x^{*}\right)\left[1+c_{2}\left(1+\gamma f^{\prime}\left(x^{*}\right)\right) e_{k}+\left(c_{2}^{2}\left(1+2 \gamma f^{\prime}\left(x^{*}\right)\right)+c_{3}\left(1+\gamma^{2} f^{\prime}\left(x^{*}\right)^{2}\right)\right) e_{k}^{2}\right. \\
& \left.+\left(-c_{2}^{3}\left(2+2 \gamma f^{\prime}\left(x^{*}\right) \gamma^{2} f^{\prime}\left(x^{*}\right)^{2}\right)+c_{2} c_{3}\left(3+8 \gamma f^{\prime}\left(x^{*}\right)+4 \gamma^{2} f^{\prime}\left(x^{*}\right)^{2}\right)+c_{4}\left(1+\gamma f^{\prime}\left(x^{*}\right)\right)^{3}\right) e_{k}^{3}\right]+\mathcal{O}\left(e_{k}^{4}\right) .
\end{aligned}
$$

Therefore, the error equation is

$$
\begin{aligned}
e_{k+1}= & -c_{2}(H(0)-1)\left(1+\gamma f^{\prime}\left(x^{*}\right)\right) e_{k}^{2}+\left[-c_{2}^{2}\left(2+2 \gamma f^{\prime}\left(x^{*}\right)+\gamma^{2} f^{\prime}\left(x^{*}\right)^{2}+H^{\prime}(0)\left(1+\gamma f^{\prime}\left(x^{*}\right)\right)^{2}\right.\right. \\
& \left.\left.-H(0)\left(3+4 \gamma f^{\prime}\left(x^{*}\right)+2 \gamma^{2} f^{\prime}\left(x^{*}\right)^{2}\right)\right)-c_{3}(H(0)-1)\left(2+3 \gamma f^{\prime}\left(x^{*}\right)+\gamma^{2} f^{\prime}\left(x^{*}\right)^{2}\right)\right] e_{k}^{3} \\
& +\left[-\frac{c_{2}^{3}}{2}\left(-8+H^{\prime \prime}(0)-10 \gamma f^{\prime}\left(x^{*}\right)-3 H^{\prime \prime}(0) \gamma f^{\prime}\left(x^{*}\right)-6 \gamma^{2} f^{\prime}\left(x^{*}\right)^{2}\right.\right. \\
& +3 H^{\prime \prime}(0) \gamma^{2} f^{\prime}\left(x^{*}\right)^{2}-2 \gamma^{3} f^{\prime}\left(x^{*}\right)^{3}+H^{\prime \prime}(0) \gamma^{3} f^{\prime}\left(x^{*}\right)^{3}+2 H(0)\left(7+11 \gamma f^{\prime}\left(x^{*}\right)+8 \gamma^{2} f^{\prime}\left(x^{*}\right)^{2}+\gamma^{3} f^{\prime}\left(x^{*}\right)^{3}\right) \\
& \left.-2 H^{\prime}(0)\left(6+13 \gamma f^{\prime}\left(x^{*}\right)+10 \gamma^{2} f^{\prime}\left(x^{*}\right)^{2}+3 \gamma^{3} f^{\prime}\left(x^{*}\right)^{3}\right)\right)+c_{2} c_{3}\left(-7-10 \gamma f^{\prime}\left(x^{*}\right)-7 \gamma^{2} f^{\prime}\left(x^{*}\right)^{2}\right. \\
& \left.-2 \gamma^{3} f^{\prime}\left(x^{*}\right)^{3}-2 H^{\prime}(0)\left(1+\gamma f^{\prime}\left(x^{*}\right)\right)^{2}\left(2+\gamma f^{\prime}\left(x^{*}\right)\right)+2 H^{\prime}(0)\left(5+9 \gamma f^{\prime}\left(x^{*}\right)+7 \gamma^{2} f^{\prime}\left(x^{*}\right)^{2}+2 \gamma^{3} f^{\prime}\left(x^{*}\right)^{3}\right)\right) \\
& \left.-c_{4}\left((H(0)-1)\left(3+6 \gamma f^{\prime}\left(x^{*}\right)+4 \gamma^{2} f^{\prime}\left(x^{*}\right)^{2}+\gamma^{3} f^{\prime}\left(x^{*}\right)^{3}\right)\right)\right] e_{k}^{4}+\mathcal{O}\left(e_{k}^{5}\right) .
\end{aligned}
$$


Setting $H(0)=1$ the error equation gets

$$
\begin{aligned}
e_{k+1}= & -c_{2}^{2}\left(H^{\prime}(0)-1\right)\left(1+\gamma f^{\prime}\left(x^{*}\right)\right)^{2} e_{k}^{3} \\
& -\frac{c_{2}}{2}\left[\left(1+\gamma f^{\prime}\left(x^{*}\right)\left(c _ { 2 } ^ { 2 } \left(6+6 \gamma f^{\prime}\left(x^{*}\right)+4 \gamma^{2} f^{\prime}\left(x^{*}\right)^{2}+H^{\prime \prime}(0)\left(1+\gamma f^{\prime}\left(x^{*}\right)\right)^{2}-2 H^{\prime}(0)(6+\right.\right.\right.\right. \\
& \left.\left.\left.\left.+7 \gamma f^{\prime}\left(x^{*}\right)+3 \gamma^{2} f^{\prime}\left(x^{*}\right)^{2}\right)\right)+2 c_{3}\left(1+\gamma f^{\prime}\left(x^{*}\right)\right)\left(-3-2 \gamma f^{\prime}\left(x^{*}\right)+2 H^{\prime}(0)\left(2+\gamma f^{\prime}\left(x^{*}\right)\right)\right)\right)\right] e_{k}^{4}+\mathcal{O}\left(e_{k}^{5}\right),
\end{aligned}
$$

and assuming also $H^{\prime}(0)=1$,

$$
e_{k+1}=-\frac{c_{2}}{2}\left(1+\gamma f^{\prime}\left(x^{*}\right)\right)^{2}\left[\left(-6+\gamma f^{\prime}\left(x^{*}\right)\left(H^{\prime \prime}(0)-2\right)+H^{\prime \prime}(0)\right) c_{2}^{2}+2 c_{3}\right] e_{k}^{4}+\mathcal{O}\left(e_{k}^{5}\right) .
$$

Let us remark that $\left(1+\gamma f^{\prime}\left(x^{*}\right)\right)$ also appears as a factor in the term of order five.

Every method of family (2) is optimal in the sense of the conjecture of Kung and Traub [17. Related to Ostrowski's efficiency index $I\left[18\right.$, the family has $I=4^{1 / 3} \approx 1.587$.

Many functions $H(t)$ satisfy the conditions of Theorem 2 , such as

1. $H(t)=1+t+\alpha t^{2}$,

2. $H(t)=\frac{1+\alpha t}{1+(\alpha-1) t}$,

3. $H(t)=\frac{\alpha+t+t^{2}}{\alpha+(1-\alpha) t}$,

where $\alpha$ is a free parameter in every case.

\section{Iterative methods with memory}

Focusing on the error equation (3), methods with memory can be obtained choosing suitable values of parameter $\gamma$. The order of convergence increases if $\gamma=-\frac{1}{f^{\prime}\left(x^{*}\right)}$, with independence of the weight function. Then the error equation becomes

$$
e_{k+1}=\left(2 c_{2}^{5}-c_{2}^{3} c_{3}\right) e_{k}^{6}+\mathcal{O}\left(e_{k}^{7}\right)
$$

so the order of convergence has increased in two units.

Nevertheless, the value of $f^{\prime}\left(x^{*}\right)$ is unknown, so it needs to be approximated. By applying Newton's interpolation polynomial of first degree, $N(t)=f\left(x_{k}\right)+f\left[x_{k}, x_{k-1}\right]\left(t-x_{k}\right), f^{\prime}\left(x^{*}\right) \approx N^{\prime}\left(x_{k}\right)=f\left[x_{k}, x_{k-1}\right]$ and, therefore, $\gamma_{k}=-\frac{1}{f\left[x_{k}, x_{k-1}\right]}$. The algorithm of the family with memory $M(\alpha)$ is summarized below:

- $x_{0}, \gamma_{0}$ are known,

- $w_{k}=x_{k}+\gamma_{k} f\left(x_{k}\right), k=0,1,2, \ldots$,

- $y_{k}=x_{k}-\frac{f\left(x_{k}\right)}{f\left[x_{k}, w_{k}\right]}$,

- $x_{k+1}=y_{k}-H\left(t_{k}\right) \frac{f\left(y_{k}\right)}{f\left[y_{k}, w_{k}\right]}$,

- $\gamma_{k}=-\frac{1}{f\left[x_{k}, x_{k-1}\right]}$.

where $\alpha$ is a disposable parameter. The order of convergence is set in the following result.

Theorem 3. Let $x^{*}$ be a simple zero of a sufficiently differentiable function $f: I \subseteq \mathbb{R} \rightarrow \mathbb{R}$ in an open interval I. If $x_{0}$ is close enough to $x^{*}$ and $\gamma_{0}$ is given, then the R-order of family $M(\alpha)$ is at least $2+\sqrt{6} \approx 4.45$ that corresponds to the positive root of polynomial $p^{2}-4 p-2$, and its error equation is

$$
e_{k+1}=\left(2 c_{2}^{5}-c_{2}^{3} c_{3}\right) e_{k}^{4} e_{k-1}^{2}+\mathcal{O}_{7}\left(e_{k}, e_{k-1}\right),
$$

where $\mathcal{O}_{7}\left(e_{k}, e_{k-1}\right)$ indicates that the sum of the exponents of $e_{k}$ and $e_{k-1}$ in the rejected terms of the development is at least $\%$. 
Proof. The expansion of $f\left(x_{k}\right)$ and $f\left(x_{k-1}\right)$ is

$$
\begin{aligned}
& f\left(x_{k}\right)=f^{\prime}\left(x^{*}\right)\left[e_{k}+c_{2} e_{k}^{2}+c_{3} e_{k}^{3}+c_{4} e_{k}^{4}+c_{5} e_{k}^{5}+c_{6} e_{k}^{6}\right]+\mathcal{O}\left(e_{k}^{7}\right), \\
& f\left(x_{k-1}\right)=f^{\prime}\left(x^{*}\right)\left[e_{k-1}+c_{2} e_{k-1}^{2}+c_{3} e_{k-1}^{3}+c_{4} e_{k-1}^{4}+c_{5} e_{k-1}^{5}+c_{6} e_{k-1}^{6}\right]+\mathcal{O}\left(e_{k-1}^{7}\right) .
\end{aligned}
$$

Since $\gamma=-\frac{1}{f\left[x_{k}, x_{k-1}\right]}$,

$$
\begin{aligned}
f\left[x_{k}, x_{k-1}\right]= & f^{\prime}\left(x^{*}\right)\left[1+c_{2} e_{k-1}+c_{3} e_{k-1}^{2}+c_{4} e_{k-1}^{3}+c_{5} e_{k-1}^{4}+c_{6} e_{k-1}^{5}\right. \\
& +\left(c_{2}+c_{3} e_{k-1}+c_{4} e_{k-1}^{2}+c_{5} e_{k-1}^{3}+c_{6} e_{k-1}^{4}\right) e_{k} \\
& +\left(c_{3}+c_{4} e_{k-1}+c_{5} e_{k-1}^{2}+c_{6} e_{k-1}^{3}\right) e_{k}^{2}+\left(c_{4}+c_{5} e_{k-1}+c_{6} e_{k-1}^{2}\right) e_{k}^{3} \\
& \left.+\left(c_{5}+c_{6} e_{k-1}\right) e_{k}^{4}+c_{6} e_{k}^{5}\right]+\mathcal{O}_{6}\left(e_{k}, e_{k-1}\right),
\end{aligned}
$$

then,

$$
\begin{aligned}
w_{k}-x^{*}= & {\left[c_{2} e_{k-1}+\left(-c_{2}^{2}+c_{3}\right) e_{k-1}^{2}+\left(c_{2}^{3}-2 c_{2} c_{3}+c_{4}\right) e_{k-1}^{3}+\left(-c_{2}^{4}+3 c_{2}^{2} c_{3}-c_{3}^{2}-2 c_{2} c_{4}+c_{5}\right) e_{k-1}^{4}\right.} \\
& \left.+\left(c_{2}^{5}-4 c_{2}^{3} c_{3}+3 c_{2}^{2} c_{4}-2 c_{3} c_{4}+c_{2}\left(3 c_{3}^{2}-2 c_{5}\right)+c_{6}\right) e_{k-1}^{5}\right] e_{k} \\
& +\left[\left(-c_{2}^{2}+c_{3}\right) e_{k-1}+\left(2 c_{2}^{3}-3 c_{2} c_{3}+c_{4}\right) e_{k-1}^{2}+\left(-3 c_{2}^{4}+7 c_{2}^{2} c_{3}-2 c_{3}^{2}-3 c_{2} c_{4}+c_{5}\right) e_{k-1}^{3}\right. \\
& \left.+\left(4 c_{2}^{5}-13 c_{2}^{3} c_{3}+7 c_{2}^{2} c_{4}-4 c_{3} c_{4}+c_{2}\left(8 c_{3}^{2}-3 c_{5}\right)+c_{6}\right) e_{k-1}^{4}\right] e_{k}^{2} \\
& +\left[\left(c_{2}^{3}-2 c_{2} c_{3}+c_{4}\right) e_{k-1}+\left(-3 c_{2}^{4}+7 c_{2}^{2} c_{3}-2 c_{3}^{2}-3 c_{2} c_{4}+c_{5}\right) e_{k-1}^{2}\right. \\
& \left.+\left(6 c_{2}^{5}-18 c_{2}^{3} c_{3}+8 c_{2}^{2} c_{4}-5 c_{3} c_{4}+c_{2}\left(11 c_{3}^{2}-3 c_{5}\right)+c_{6}\right) e_{k-1}^{3}\right] e_{k}^{3} \\
& +\left[\left(-c_{2}^{4}+3 c_{2}^{2} c_{3}-c_{3}^{2}-2 c_{2} c_{4}+c_{5}\right) e_{k-1}+\left(4 c_{2}^{5}-13 c_{2}^{3} c_{3}+7 c_{2}^{2} c_{4}-4 c_{3} c_{4}+c_{2}\left(8 c_{3}^{2}-3 c_{5}\right)+c_{6}\right) e_{k-1}^{2}\right] e_{k}^{4} \\
& +\left[\left(c_{2}^{5}-4 c_{2}^{3} c_{3}+3 c_{2}^{2} c_{4}-2 c_{3} c_{4}+c_{2}\left(3 c_{3}^{2}-2 c_{5}\right)+c_{6}\right) e_{k-1}\right] e_{k}^{5}+\mathcal{O}_{7}\left(e_{k}, e_{k-1}\right)
\end{aligned}
$$

The divided difference is

$$
\begin{aligned}
f\left[x_{k}, w_{k}\right]= & f^{\prime}\left(x^{*}\right)\left[1+c_{2}\left(1+c_{2} e_{k-1}+\left(-c_{2}^{2}+c_{3}\right) e_{k-1}^{2}+\left(c_{2}^{3}-2 c_{2} c_{3}+c_{4}\right) e_{k-1}^{3}\right.\right. \\
& \left.-\left(c_{2}^{3}-3 c_{2}^{2} c_{3}+c_{3}^{2}+2 c_{2} c_{4}-c_{5}\right) e_{k-1}^{4}\right) e_{k}+\left(c_{3}+\left(-c_{3}^{2}+2 c_{2} c_{3}\right) e_{k-1}\right. \\
& \left.+\left(2 c_{2}^{4}-3 c_{2}^{2} c_{3}+c_{3}^{2}+c_{2} c_{4}\right) e_{k-1}^{2}+\left(-3 c_{2}^{5}+6 c_{2}^{3} c_{3}-3 c_{2}^{2} c_{4}+c_{3} c_{4}+c_{2}\left(-2 c_{3}^{2}+c_{5}\right)\right) e_{k-1}^{3}\right) e_{k}^{2} \\
& +\left(c_{4}+\left(c_{2}^{4}-3 c_{2}^{2} c_{3}+c_{3}^{2}+2 c_{2} c_{4}\right) e_{k-1}+\left(-3 c_{2}^{5}+7 c_{2}^{3} c_{3}-3 c_{2}^{2} c_{4}+2 c_{3} c_{4}+c_{2}\left(-3 c_{3}^{2}+c_{5}\right)\right) e_{k-1}^{2}\right) e_{k}^{3} \\
& \left.+\left(c_{5}+\left(-c_{2}^{5}+4 c_{2}^{3} c_{3}-3 c_{2}^{2} c_{4}+2 c_{3} c_{4}+c_{2}\left(-3 c_{3}^{2}+2 c_{5}\right)\right) e_{k-1}\right) e_{k}^{4}+c_{6} e_{k}^{5}\right]+\mathcal{O}_{6}\left(e_{k}, e_{k-1}\right),
\end{aligned}
$$

so the first step results in

$$
\begin{aligned}
y-x^{*}= & {\left[c_{2}\left(c_{2}+\left(-c_{2}^{2}+c_{3}\right) e_{k-1}+\left(c_{2}^{3}-2 c_{2} c_{3}+c_{4}\right) e_{k-1}^{2}-\left(c_{2}^{4}-3 c_{2}^{2} c_{3}+c_{3}^{2}+2 c_{2} c_{4}-c_{5}\right) e_{k-1}^{3}\right)\right.} \\
& +\left(-2 c_{2}^{3}+2 c_{2} c_{3}+\left(2 c_{2}^{4}-4 c_{2}^{2} c_{3}+c_{3}^{2}+c_{2} c_{4}\right) e_{k-1}+\left(-2 c_{2}^{5}+6 c_{2}^{3} c_{3}-4 c_{2}^{2} c_{4}+c_{3} c_{4}+c_{2}\left(-2 c_{3}^{2}+c_{5}\right)\right) e_{k-1}^{2}\right) e_{k} \\
& +\left(3 c_{2}^{4}-6 c_{2}^{2} c_{3}+c_{3}^{2}+2 c_{2} c_{4}+\left(-2 c_{2}^{5}+8 c_{2}^{3} c_{3}-4 c_{2}^{2} c_{4}+2 c_{3} c_{4}+c_{2}\left(-5 c_{3}^{2}+c_{5}\right) e_{k-1}\right)\right) e_{k}^{2} \\
& \left.+2\left(-c_{2}^{5}+6 c_{2}^{3} c_{3}-3 c_{2}^{2} c_{4}+c_{3} c_{4}+c_{2}\left(-3 c_{3}^{2}+c_{5}\right)\right) e_{k}^{3}\right] e_{k}^{2} e_{k-1}+\mathcal{O}_{7}\left(e_{k}, e_{k-1}\right) .
\end{aligned}
$$

The next divided difference is

$$
\begin{aligned}
f\left[y_{k}, w_{k}\right]= & f^{\prime}\left(x^{*}\right)\left[1+c_{2}\left(c_{2}+\left(-c_{2}^{2}+c_{3}\right) e_{k-1}+\left(c_{2}^{3}-2 c_{2} c_{3}+c_{4}\right) e_{k-1}^{2}\right) e_{k} e_{k-1}\right. \\
& \left.+c_{2}\left(c_{3}+\left(c_{2}^{3}-c_{2} c_{3}+c_{4}\right) e_{k-1}\right) e_{k}^{2} e_{k-1}+\left(-c_{2}^{4}+c_{2} c_{4}\right) e_{k}^{3}\right]+\mathcal{O}_{5}\left(e_{k}, e_{k-1}\right),
\end{aligned}
$$

then, the error equation is

$$
e_{k+1}=\left(2 c_{2}^{5}-c_{2}^{3} c_{3}\right) e_{k}^{4} e_{k-1}^{2}+\mathcal{O}_{7}\left(e_{k}, e_{k-1}\right) .
$$

Since the lower term of the error equation is $\left(2 c_{2}^{5}-c_{2}^{3} c_{3}\right) e_{k-1}^{2} e_{k}^{4}$, applying Theorem 1 , the powers of $e_{k}$ and $e_{k-1}$ are 4 and 2, respectively, so the polynomial whose real roots give the R-order of the method is $p^{2}-4 p+2$ and the order of the method is, at least, $2+\sqrt{6}$. 
Although the order of convergence of the methods with memory does not depend on the selected weight function, the analysis of the stability is different depending on $H(t)$. So, from now on, we use the tools of real discrete dynamics on the subclass described by the previous algorithm using $H(t)=1+t+\alpha t^{2}$.

\section{Dynamical analysis}

The dynamics of the family of iterative methods with memory $M(\alpha)$ is analyzed in this section. Some fundamentals about memory dynamics are introduced below. Further information can be found in [19, 20].

\subsection{Fundamentals on dynamics of methods with memory}

The standard form of an iterative method with memory of first order is

$$
x_{k+1}=\phi\left(x_{k}, x_{k-1}\right), \quad k \geq 1 \text {, }
$$

where $x_{0}$ and $x_{1}$ are the initial estimations. As a function defined from $\mathbb{R}^{2}$ to $\mathbb{R}$ can not have fixed points, we define an auxiliary vectorial function $\Phi$ by means of

$$
\Phi\left(x_{k-1}, x_{k}\right)=\left(x_{k}, x_{k+1}\right)=\left(x_{k}, \phi\left(x_{k-1}, x_{k}\right)\right), \quad k=1,2, \ldots
$$

Then, $\left(x_{k-1}, x_{k}\right)$ is a fixed point of $\Phi$ if $\Phi\left(x_{k-1}, x_{k}\right)=\left(x_{k-1}, x_{k}\right)$ and, consequently, $x_{k+1}=x_{k}$ and $x_{k}=x_{k-1}$. Therefore, the discrete dynamical system $\Phi: \mathbb{R}^{2} \rightarrow \mathbb{R}^{2}$ is defined such that

$$
\Phi(\vec{x})=\Phi(z, x)=(x, \phi(z, x)),
$$

where $\phi$ is the operator of the iterative scheme with memory. Below some basic definitions are recalled.

The orbit of a point $\vec{x}_{0}$ is defined as the set $\left\{\vec{x}_{0}, \Phi\left(\vec{x}_{0}\right), \Phi^{2}\left(\vec{x}_{0}\right), \ldots, \Phi^{n}\left(\vec{x}_{0}\right), \ldots\right\}$. A point $\vec{x}_{0}=(z, x)_{0}$ is a fixed point $\vec{x}_{0}^{F}=(z, x)_{0}^{F}$ of $\Phi$ if $z=x$ and $x=\phi(z, x)$. If a fixed point $\vec{x}^{F}$ of operator $\Phi$ is different from $(r, r)$, where $r$ satisfies $f(r)=0$, it is called strange fixed point. A point $\vec{x}_{T}$ is T-periodic if $\Phi^{T}\left(\vec{x}_{T}\right)=\vec{x}_{T}$ and $\Phi^{t}\left(\vec{x}_{T}\right) \neq \overrightarrow{x_{T}}$, for $t<T$.

The stability of a periodic point $\vec{x}_{T}$ is defined from its asymptotical behavior. For this purpose, Theorem 4 of 21] is shown.

Theorem 4. Let $\Phi$ from $\mathbb{R}^{n}$ to $\mathbb{R}^{n}$ be $\mathcal{C}^{2}$. Assume $\vec{x}_{T}$ is T-periodic. Let $\lambda_{1}, \lambda_{2}, \ldots, \lambda_{n}$ be the eigenvalues of $\Phi^{\prime}\left(\vec{x}_{T}\right)$. Then,

1. If all the eigenvalues $\lambda_{j}$ have $\left|\lambda_{j}\right|<1$, then $\vec{x}_{T}$ is attracting.

2. If one eigenvalue $\lambda_{j_{0}}$ has $\left|\lambda_{j_{0}}\right|>1$, then $\vec{x}_{T}$ is unstable, that is, repelling or saddle.

3. If all the eigenvalues $\lambda_{j}$ have $\left|\lambda_{j}\right|>1$, then $\vec{x}_{T}$ is repelling.

In addition,

(a) If all the eigenvalues $\lambda_{j}$ have $\left|\lambda_{j}\right| \neq 1$, the T-periodic point is hyperbolic.

(b) If there exist an eigenvalue $\lambda_{i}:\left|\lambda_{i}\right|<1$ and another eigenvalue $\lambda_{j}:\left|\lambda_{j}\right|>1$, then the hyperbolic point is recalled as saddle point.

(c) If all the eigenvalues are equal to zero the T-periodic point is superattracting.

A critical point $\vec{x}^{C}$ satisfies $\operatorname{det}\left(\Phi^{\prime}\left(\vec{x}^{C}\right)\right)=0$. The basin of attraction of a T-periodic point $\vec{x}^{*}$, is defined as the set of pre-images of any order such that

$$
\mathcal{A}\left(\vec{x}^{*}\right)=\left\{\vec{x}_{0} \in \mathbb{R}^{n}: \Phi^{m}\left(\vec{x}_{0}\right) \rightarrow \vec{x}^{*}, m \rightarrow \infty\right\} .
$$

The drawing tool to represent the basins of attraction is the dynamical plane [22, 23]. For real dynamics with memory, the horizontal axis is devoted to the current iteration $x_{k}$ while the vertical one represents the last iteration $x_{k-1}$. The method is analysed over a mesh of values of $x_{k}$ and $x_{k-1}$ as initial guesses. Each attracting point is represented with a non-black colour. If the orbit of these initial guesses tends to an attracting fixed point, the initial guess $\left(x_{k}, x_{k-1}\right)$ is depicted in the corresponding colour; otherwise, the initial guess is depicted in black.

Moreover, there exist other ways to represent the real dynamics, such as the bifurcation diagram (also known as Feigenbaum diagram) or the convergence plane [24]. 


\subsection{Dynamics of $M(\alpha)$ on quadratic polynomials}

The dynamical behavior of the family $M(\alpha)$ is studied when it is applied on quadratic polynomials $f(x)=$ $x^{2}+\{-1,0,1\}$ as it is usual (see [25]).

When $M(\alpha)$ is applied on $f(x)=x^{2}-1$, the fixed point operator results in

$$
M_{-1}(z, x, \alpha)=\frac{N_{15}(z, x, \alpha)}{D_{14}(x, z, \alpha)},
$$

where $N_{15}(x, z, \alpha)$ and $D_{14}(z, x, \alpha)$ stand for polynomials of degrees 15 and 14, respectively, that depend on the variables $z$ and $x$ and the parameter $\alpha$.

We define the operator $\Phi_{-1}: \mathbb{R}^{2} \rightarrow \mathbb{R}^{2}$ as

$$
\Phi_{-1}(z, x)=\left(x, M_{-1}(z, x, \alpha)\right) .
$$

The fixed points of this vectorial rational function can be calculated by solving the system formed by equations $x=M_{-1}(z, x, \alpha)$ and $z=x$. In this way, it is obtained that there exist 7 real fixed points of $\Phi_{-1}(z, x)$ :

- $(z, x)_{1,2}^{F}=(\mp 1, \mp 1)$, whose components are the roots of the polynomial.

- $(z, x)_{3}^{F}=(0,0)$.

- The equal components of $(z, x)_{4-7}^{F}$ are real roots of the polynomial $p(s)=s^{12}(\alpha+495)+s^{10}(1266-6 \alpha)+$ $s^{8}(15 \alpha+1381)+s^{6}(716-20 \alpha)+s^{4}(15 \alpha+201)+s^{2}(34-6 \alpha)+\alpha+3$ for some values of parameter $\alpha .(z, x)_{4,5}^{F}$ are real fixed points for $\alpha<-3$, while $(z, x)_{6,7}^{F}$ are real fixed points for $\alpha<-495$.

In order to deduce the stability of the fixed points, we calculate the Jacobian matrix associated to $\Phi_{-1}$,

$$
\Phi_{-1}^{\prime}(z, x)=\left[\begin{array}{cc}
0 & 1 \\
\frac{\partial M_{-1}(z, x, \alpha)}{\partial z} & \frac{\partial M_{-1}(z, x, \alpha)}{\partial x}
\end{array}\right],
$$

and its eigenvalues are denoted by $\lambda_{1}, \lambda_{2}$.

For $(z, x)_{1,2}^{F}=(\mp 1, \mp 1), \lambda_{1}=\lambda_{2}=0$, so the fixed points are superattracting. For $(z, x)_{3}^{F}=(0,0), \lambda_{1}=$ $\frac{4-\alpha-\sqrt{28+12 \alpha+\alpha^{2}}}{2}$ and $\lambda_{2}=\frac{4-\alpha+\sqrt{28+12 \alpha+\alpha^{2}}}{2}$, so the absolute values of $\lambda_{1}$ and $\lambda_{2}$ are both lower than 1 for $\alpha \in$ $(-4,-3)^{2}$ and, therefore, $(z, x)_{3}^{F}$ is only attracting in this region of $\alpha$. From a similar study we can conclude that $(z, x)_{4-7}^{F}$ are unstable for every value of $\alpha$. Table 1 summarizes the behaviour of every fixed point.

\begin{tabular}{|c|c|c|c|c|}
\hline$\alpha$ & $(z, x)_{1,2}^{F}$ & $(z, x)_{3}^{F}$ & $(z, x)_{4,5}^{F}$ & $(z, x)_{6,7}^{F}$ \\
\hline$<-495$ & $\mathrm{~A}$ & $\mathrm{R}$ & $\mathrm{R}$ & $\mathrm{S}$ \\
$\in(-495,-4)$ & $\mathrm{A}$ & $\mathrm{R}$ & $\mathrm{S}$ & $\mathrm{X}$ \\
$\in(-4,-3)$ & $\mathrm{A}$ & $\mathrm{A}$ & $\mathrm{S}$ & $\mathrm{X}$ \\
$\in(-3,720)$ & $\mathrm{A}$ & $\mathrm{S}$ & $\mathrm{X}$ & $\mathrm{X}$ \\
$>720$ & $\mathrm{~A}$ & $\mathrm{~S}$ & $\mathrm{X}$ & $\mathrm{X}$ \\
\hline
\end{tabular}

Table 1: Behaviour of fixed points of $\Phi_{-1}(z, x, \alpha)$. A: attracting, R: repelling, S: saddle, X: not a real fixed point.

Now, we present the bifurcation diagrams of the map associated to $M(\alpha)$ family on quadratic polynomial $f(x)$, by using as a starting point each one of the strange fixed points of the map and observing the ranges of the parameter $\alpha$ where changes of stability or other behaviors happen. This allows us to check the studied dependence of the stability of these points on the parameter.

To draw Feigenbaum diagrams, 700 elements of the orbit of each strange fixed point are calculated, plotting the last 200, for each value of parameter $\alpha$ (after a partition of the analyzed interval in 3000 subintervals).

The bifurcation diagram of the strange fixed points is shown below. This diagram represents the possible orbits of a point in the neighborhood of the strange point. 


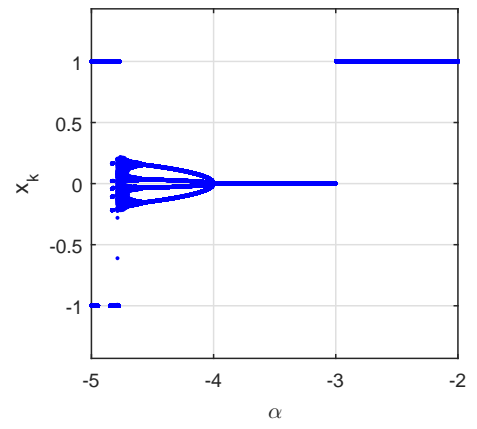

(a) $(z, x)_{3}^{F}$

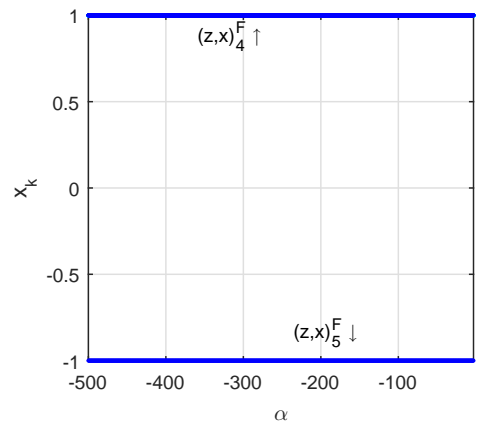

(b) $(z, x)_{4,5}^{F}$

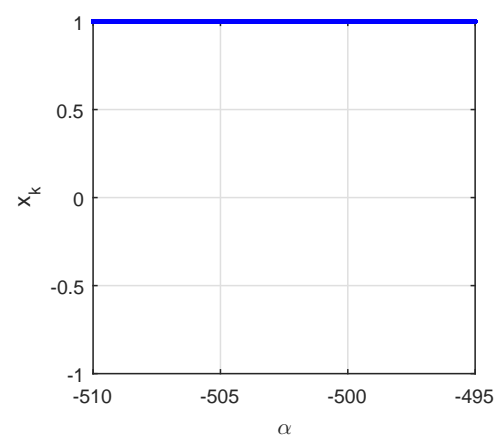

(c) $(z, x)_{6,7}^{F}$

Figure 1: Bifurcation diagram of $(z, x)_{3-7}^{F}$

Figure 1 (a) shows that $(z, x)_{3}^{F}=(0,0)$ is an attracting point for $\alpha \in(-4,-3)$, as mentioned before. Moreover, the fixed point bifurcates into a 4-periodic orbit when $\alpha<-4$ and when $\alpha$ is closed to -5 is bifurcates again and a chaotic region is observed. Figures 1 (b) and (c) show that $(z, x)_{4-7}^{F}$ are unstable, since every point tend to one of the roots for all the analyzed values of $\alpha$ for which they are fixed points.

The dynamical planes represent a single method of the complete family, selected by choosing a value of $\alpha$. Some dynamical planes are represented in Figure 2, Each value of $\alpha$ is selected in a region where behaviour of the fixed points is different. Orange points represent convergence to $(z, x)_{1}^{F}=(-1,-1)$, while blue ones tend to $(z, x)_{2}^{F}=(1,1)$. Green points represent the basin of attraction of $(z, x)_{3}^{F}=(0,0)$. Black points are reserved for those that do not converge to any of the previous basins of attraction. Each dynamical plane is obtained by applying a mesh of $300 \times 300$ initial guesses. Further information about the generation of dynamical planes can be found in [22.

The dynamical planes confirm the expected behaviour. Figures 2 (a,b,e,f) only have convergence to the fixed points corresponding to the roots of the polynomial. In Figure 2(c), a region without convergence to any root can be found. As deduced from Figure 1, these points remain in a 4-period orbit, as the yellow orbit of Figure 2 describes. Finally, Figure 2(d) shows a case in which the origin is an attracting fixed point.

Applying $M(\alpha)$ over the polynomial $f(x)=x^{2}$, the fixed point operator is

$$
M_{0}(z, x, \alpha)=\frac{x z\left(x^{5}+10 x^{4} z+39 x^{3} z^{2}+75 x^{2} z^{3}-(\alpha-72) x z^{4}-(\alpha-28) z^{5}\right)}{(x+2 z)^{5}(2 x+3 z)} .
$$

In this case, we analyze the existence of fixed points of $\Phi_{0}(z, x)=\left(x, M_{0}(z, x, \alpha)\right)$ in a similar way as it has been done for $\Phi_{-1}(z, x)$. Firstly, it can be proven that $(z, x)_{1}^{F}=(0,0)$ is a fixed point as is the root of $f(x)$. On the other hand, we can prove that, for $\alpha=-495$, there exist a line of critical points $(z, x)_{2}^{F}=(\tau, \tau), \forall \tau \in \mathbb{R}$.

The dynamical behaviour is analysed from $\Phi_{0}(z, x)=\left(x, M_{0}(z, x)\right)$. The Jacobian matrix of a generic fixed point $(z, x)^{F}=(t, t)$ is

$$
\Phi_{0}^{\prime}(t, t, \alpha)=\left[\begin{array}{cc}
0 & 1 \\
\frac{1215-47 \alpha}{18225} & \frac{2160+17 \alpha}{18225}
\end{array}\right],
$$

whose eigenvalues are $\lambda_{1}=\frac{-\sqrt{289 \alpha^{2}-3352860 \alpha+93239100}+17 \alpha+2160}{36450}$ and $\lambda_{2}=\frac{\sqrt{289 \alpha^{2}-3352860 \alpha+93239100}+17 \alpha+2160}{36450}$. If we analyze the values of $\alpha$ such that $\left|\lambda_{1}\right|<1$ and $\left|\lambda_{2}\right|<1$, we obtain the open interval $\alpha \in\left(-\frac{36585}{32}, \frac{19440}{47}\right)$ where every fixed point of $\Phi_{0}(z, x)$ is attracting.

Figure 3 illustrates the different behaviours. In Fig. 3(a), $(z, x)_{2}^{F}=(\tau, \tau)$ and $\alpha=-495$; the eigenvalues of these strange fixed points are $\lambda_{1}=-1.34321, \lambda_{2}=1$, so they are not hyperbolic and their behavior cannot be predicted. Figure 3(b) show the attracting behaviour of $(z, x)_{1}^{F}$; as this fixed point correspond to a multiple root, the convergence is linear, as it can be deduced from the dark orange appearing in the dynamical plane. 


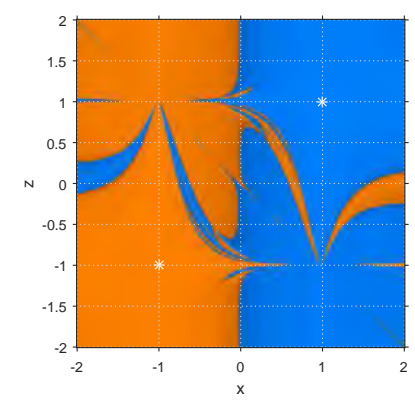

(a) $\alpha=-550$

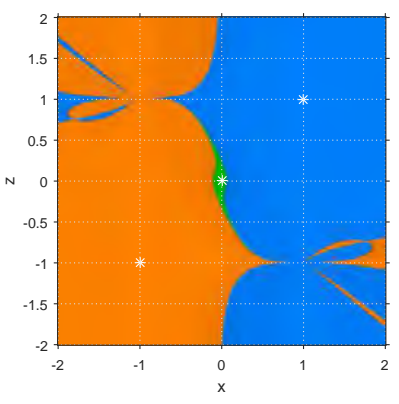

(d) $\alpha=-3.3$

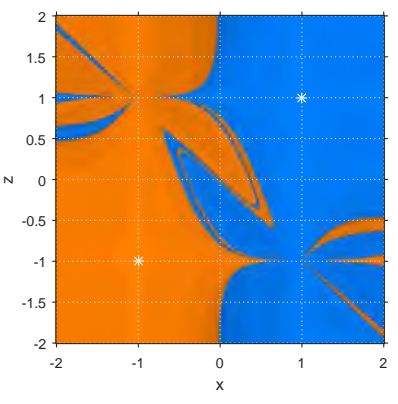

(b) $\alpha=-20$

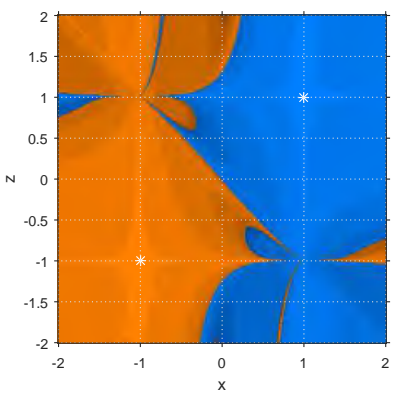

(e) $\alpha=10$

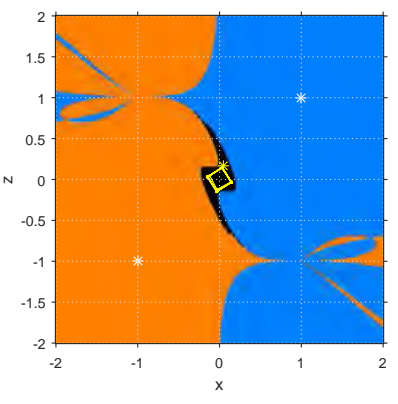

(c) $\alpha=-4.5$

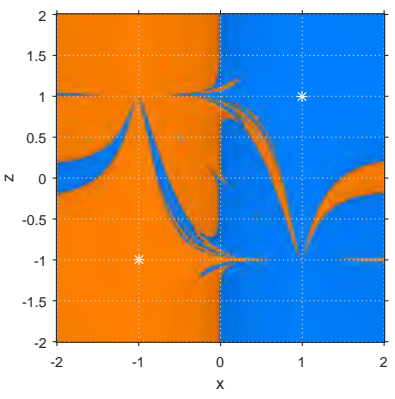

(f) $\alpha=800$

Figure 2: Dynamical planes of single members of the family $M_{-1}(z, x, \alpha)$.

Finally, the study is completed applying $M(\alpha)$ on the polynomial $f(x)=x^{2}+1$, whose associate multidimensional rational function is $\Phi_{1}(z, x)=\left(x, M_{1}(z, x, \alpha)\right)$, where

$$
M_{1}(z, x, \alpha)=\frac{N_{15}(z, x, \alpha)}{D_{14}(z, x, \alpha)}
$$

where, as in the $f(x)=x^{2}-1$ case, $N_{15}(z, x, \alpha)$ and $D_{14}(z, x, \alpha)$ stand for polynomials of degrees 15 and 14 , respectively, that depend on the variables $z$ and $x$, and the parameter $\alpha$.

As the roots of $f(x)$ are complex, only strange fixed points of $\Phi_{1}(z, x)$ can appear as they must be real. $(z, x)_{1}^{F}=(0,0)$ is fixed in the complete domain of $\alpha .(z, x)_{2-5}^{F}$ are the real roots of $q(t)=t^{12}(\alpha+495)+t^{10}(6 \alpha-$ 1266) $+t^{8}(15 \alpha+1381)+t^{6}(20 \alpha-716)+t^{4}(15 \alpha+201)+t^{2}(6 \alpha-34)+\alpha+3$, and they are real fixed points only in some regions of $\alpha$. Finally, for $\alpha=-3,(z, x)_{6,7}^{F}=(\mp 1.17384, \mp 1.17384)$.

The eigenvalues $\lambda_{1}$ and $\lambda_{2}$ of the Jacobian matrix $\Phi_{1}^{\prime}(z, x)$ determine the behaviour of each of these fixed points. For $(z, x)_{1}^{F}=(0,0)$, the eigenvalues are $\lambda_{1}=\frac{4+\alpha-\sqrt{28+12 \alpha+\alpha^{2}}}{2}$ and $\lambda_{2}=\frac{4+\alpha+\sqrt{28+12 \alpha+\alpha^{2}}}{2}$. The absolute value of both eigenvalues is lower than one for $\alpha \in(-4,-3)$. Related to $(z, x)_{2}^{F}$, it is saddle for the values of $\alpha$ in which it is a fixed point. The stability of $(z, x)_{3}^{F}$ is attracting for $\alpha$ in the interval $(-3,-2.493)$, being repulsive or saddle in the rest of its domain as fixed point. However, the other two real roots of $q(t),(z, x)_{4,5}^{F}$, are attracting for $\alpha \in(-3,-2.493)$. Finally, the eigenvalues of $(z, x)_{6,7}^{F}$ are $\lambda_{1}=14.75$ and $\lambda_{2}=-0.4$, so they are saddle. Table 2 collects all the information.

Figure 4 represents the bifurcation diagrams of the attracting strange fixed points. As can be observed, the orbits tend to 0 for $\alpha \in(-4,-3)$ and it bifurcates into two of the attracting strange fixed points for $\alpha \in(-3,-2.493)$, verifying the analytical study. We can also find periodical orbits around $\alpha=-2.4$ and chaotic behaviour from $\alpha=-2.3$ forward. The bifurcation diagram of $(z, x)_{3}^{F}$ is similar to that of $(z, x)_{4,5}^{F}$ and, therefore, it is omitted.

Once we have computed a set of dynamical planes, we only show three of them that behaves in a different way, 


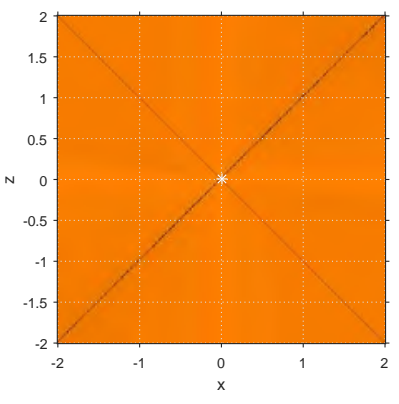

(a) $\alpha=-495$

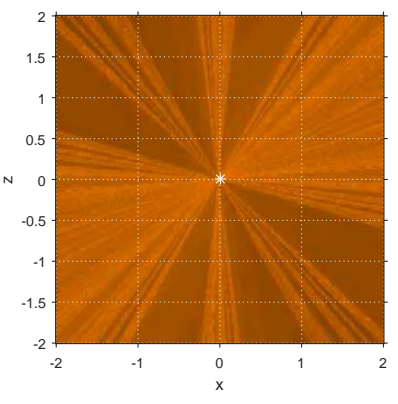

(b) $\alpha=100$

Figure 3: Dynamical planes of single members of the family $M_{0}(z, x, \alpha)$.

\begin{tabular}{|c|c|c|c|c|c|c|}
\hline$\alpha$ & $(z, x)_{1}^{F}$ & $(z, x)_{2}^{F}$ & $(z, x)_{3}^{F}$ & $(z, x)_{4}^{F}$ & $(z, x)_{5}^{F}$ & $(z, x)_{6,7}^{F}$ \\
\hline$\leq-495$ & $\mathrm{R}$ & $\mathrm{X}$ & $\mathrm{X}$ & $\mathrm{X}$ & $\mathrm{X}$ & $\mathrm{X}$ \\
$\in(-495,-5-2 \sqrt{5})$ & $\mathrm{R}$ & $\mathrm{S}$ & $\mathrm{S}$ & $\mathrm{X}$ & $\mathrm{X}$ & $\mathrm{X}$ \\
$=-5-2 \sqrt{5}$ & $\mathrm{R}$ & $\mathrm{X}$ & $\mathrm{X}$ & $\mathrm{X}$ & $\mathrm{X}$ & $\mathrm{X}$ \\
$\in(-5-2 \sqrt{5},-4)$ & $\mathrm{R}$ & $\mathrm{S}$ & $\mathrm{S}$ & $\mathrm{X}$ & $\mathrm{X}$ & $\mathrm{X}$ \\
$\in[-4,-3)$ & $\mathrm{A}$ & $\mathrm{S}$ & $\mathrm{S}$ & $\mathrm{X}$ & $\mathrm{X}$ & $\mathrm{X}$ \\
$=-3$ & $\mathrm{~S}$ & $\mathrm{X}$ & $\mathrm{X}$ & $\mathrm{X}$ & $\mathrm{X}$ & $\mathrm{S}$ \\
$\in(-3,-2.493]$ & $\mathrm{S}$ & $\mathrm{S}$ & $\mathrm{A}$ & $\mathrm{A}$ & $\mathrm{A}$ & $\mathrm{X}$ \\
$\in(-2.493,-1.91)$ & $\mathrm{S}$ & $\mathrm{S}$ & $\mathrm{R}$ & $\mathrm{R}$ & $\mathrm{S}$ & $\mathrm{X}$ \\
$\in(-1.91,-5+2 \sqrt{5})$ & $\mathrm{S}$ & $\mathrm{S}$ & $\mathrm{S}$ & $\mathrm{S}$ & $\mathrm{S}$ & $\mathrm{X}$ \\
$=-5+2 \sqrt{5}$ & $\mathrm{~S}$ & $\mathrm{~S}$ & $\mathrm{X}$ & $\mathrm{X}$ & $\mathrm{S}$ & $\mathrm{X}$ \\
$\in(-5+2 \sqrt{5}, 0)$ & $\mathrm{S}$ & $\mathrm{S}$ & $\mathrm{S}$ & $\mathrm{S}$ & $\mathrm{S}$ & $\mathrm{X}$ \\
$\geq 0$ & $\mathrm{~S}$ & $\mathrm{X}$ & $\mathrm{X}$ & $\mathrm{X}$ & $\mathrm{X}$ & $\mathrm{X}$ \\
\hline
\end{tabular}

Table 2: Behaviour of fixed points of $\Phi_{1}(z, x)$. A: attracting, R: repelling, S: saddle, X: not a fixed point.

as deduced from Table 2. These behaviours are attraction to $(z, x)_{1}^{F}$, attraction to $(z, x)_{3,4}^{F}$, and no attraction, as Figures 5(a,b,c) show, respectively. Related to the bifurcation diagram of Fig. 4 , we have chosen a value of $\alpha$ in which a periodic orbit can be found, as the yellow path of Fig. 5 (c) shows.

A very small region of convergence to $(0,0)$ can be observed in Figure 5(a), meanwhile a pair of periodic orbits can be seen in Figure 5(c) for $\alpha=-2.4$.

\section{Numerical performance}

Finally, we introduce the numerical experiment obtained by applying some new and presented methods on the following nonlinear functions:

- $f_{1}(x)=\sin x-x^{2}+1$,

- $f_{2}(x)=x^{2}-\exp (x)-3 x+2$,

- $f_{3}(x)=\cos x-x$,

- $f_{4}(x)=(x-1)^{3}-1$,

- $f_{5}(x)=x^{3}-10$,

- $f_{6}(x)=\cos x-x \exp (x)+x^{2}$, 


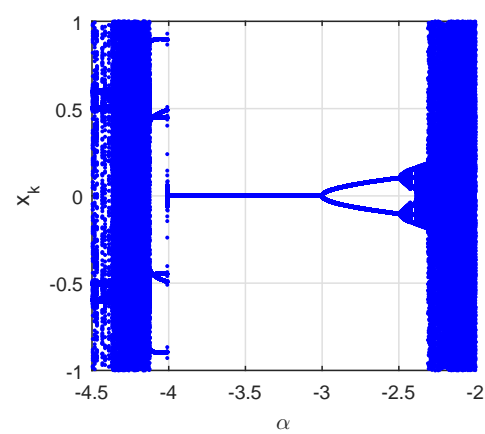

(a) $(z, x)_{1}^{F}$

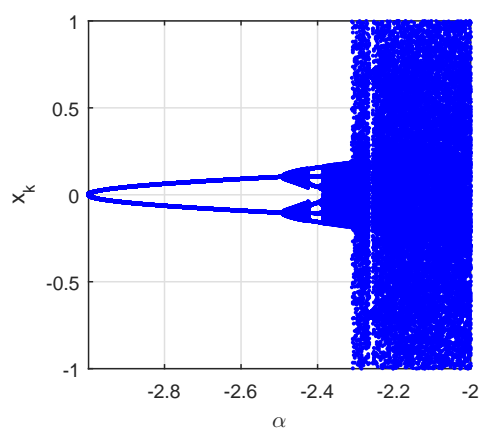

(b) $(z, x)_{4,5}^{F}$

Figure 4: Bifurcation diagrams of $(z, x)_{1,4,5}^{F}$.

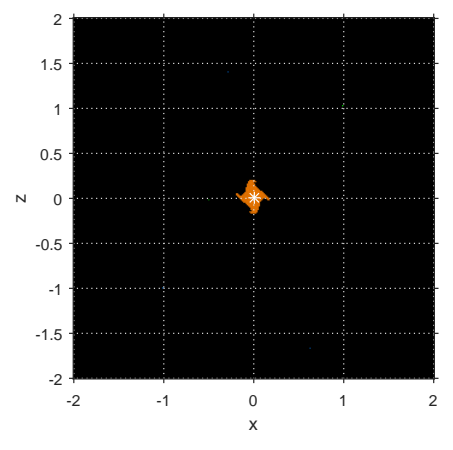

(a) $\alpha=-3.5$

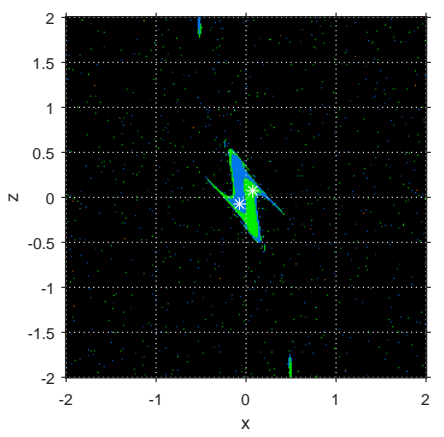

(b) $\alpha=-2.75$

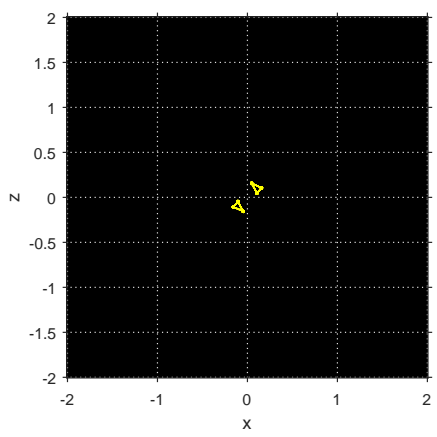

(c) $\alpha=-2.4$

Figure 5: Dynamical planes of single members of the family $M_{1}(z, x, \alpha)$.

- $f_{7}(x)=x^{3}+4 x^{2}-10$,

- $f_{8}(x)=\arctan (x)$.

For the sake of comparison, we apply methods without and with memory on the same functions. On the one hand, the schemes without memory are Newton', Ostrowski' and Jarratt's methods, denoted by NEW, OST and JAR, respectively. On the other hand, the methods with memory are the equations (15) and (21) of [7]

$$
\begin{aligned}
y_{k} & =x_{k}-\frac{\gamma_{k} f^{2}\left(x_{k}\right)}{f\left(x_{k}+\gamma_{k} f\left(x_{k}\right)\right)-f\left(x_{k}\right)} \\
x_{k+1} & =y_{k}-h\left(s_{k}, v_{k}\right) \frac{\gamma_{k} f\left(x_{k}\right) f\left(y_{k}\right)}{f\left(x_{k}+\gamma_{k} f\left(x_{k}\right)\right)-f\left(x_{k}\right)}
\end{aligned}
$$

where the accelerating parameter is $\gamma_{k}=f\left[x_{k}, x_{k-1}\right]$ and the weight functions are

- $h_{1}(s, v)=\frac{1+s}{1+v}$,

- $h_{2}(s, v)=\frac{1}{(1+s)(1+v)}$,

- $h_{3}(s, v)=1+s+v+v^{2}$,

- $h_{4}(s, v)=s+\frac{1}{1-v}$, 
where $s=f(y) / f(x)$ and $v=f(y) / f(x+\gamma f(x))$. These methods are denoted by PDP1, PDP2, PDP3 and PDP4, where the final number stands for the applied weight function.

The numerical analysis is performed of the introduced family is analyzed for the members $M(-1), M(0.5)$, $M(-495)$ and $M(720)$, whose notations are CCT1, CCT2, CCT3, and CCT4, respectively. The election of the values of $\alpha$ is related to their dynamical behaviour. On the one hand, for $\alpha \in\{-1,0.5\}$ the fixed points associated to the roots of the polynomial are attracting. On the other hand, values $\alpha \in\{-495,720\}$ are bifurcation points, wherein the behaviour is modified.

Tables 3 5 gathers the information of the numerical experience. The analysis is performed by using variable precision arithmetics with 2000 digits of mantissa in Matlab2015a. The stopping criterion is $\left|f\left(x_{k}\right)\right|<10^{-500}$. For methods with memory, the initial guesses are $x_{-1}=x_{0}+0.05$ and $x_{0}$. The data collected in Tables 35 are the difference between the two last iterations $\left|x_{k}-x_{k-1}\right|$, the evaluation of the function in the last iteration $\left|f\left(x_{k}\right)\right|$, the number of iterations iter and the approximated computational order of convergence $A C O C$ [26, whose expression is

$$
A C O C=\frac{\ln \left(\frac{\left|x_{k+1}-x_{k}\right|}{\left|x_{k}-x_{k-1}\right|}\right)}{\ln \left(\frac{\left|x_{k}-x_{k-1}\right|}{\left|x_{k-1}-x_{k-2}\right|}\right)} .
$$

ACOC is defined as a vector. The value of ACOC collected in the numerical results refers to the final value if the vector is stable.

The numerical results uphold both the analysis of the convergence and the dynamical study. CCT1 and CCT2 show better results in terms of number of iterations than CCT3 and CCT4, since they are related to stable and unstable values of $\alpha$, respectively. Furthermore, the performance of the methods with stable values of $\alpha$ show a similar behavior to PDP1 to PDP4. Finally, the with-memory methods obtain the solution of the nonlinear equation in less number of iterations than the without-memory methods, as expected.

Acknowledgement: The authors would like to thank the anonymous reviewers for their useful comments and suggestions.

[1] J. F. Steffensen, Remarks on iteration, Scandinavian Actuarial Journal 16 (1993) 64-72.

[2] J. F. Traub, Iterative methods for the solution of equations, Prentice Hall, 1964.

[3] B. Neta, A new family of high order methods for solving equations, International Journal of Computer Mathematics 14 (1983) 191-195.

[4] X. Wu, A new continuation Newton-like method and its deformation, Applied Mathematics and Computation 112 (2000) 75-78.

[5] X. Wang, T. Zhang, A new family of Newton-type iterative methods with and without memory for solving nonlinear equations, Calcolo 51 (2014) 1-15.

[6] M. S. Petković, S. Illić, J. Džunic, Derivative free two-point methods with and without memory for solving nonlinear equations, Applied Mathematics and Computation 217 (2010) 1887-1895.

[7] M. S. Petković, J. Džunic, L. D. Petković, A family of two-point methods with memory for solving nonlinear equations, Applicable Analysis and Discrete Mathematics 5 (2011) 298-317.

[8] J. Džunic, M. S. Petković, L. D. Petković, Three-point methods with and without memory for solving nonlinear equations, Applied Mathematics and Computation 218 (2012) 4917-4927.

[9] J. Džunic, M. S. Petković, On generalized biparametric multipoint root finnding methods with memory, Journal of Computer and Applied Mathematics 255 (2014) 362-375.

[10] T. Lotfi, F. Soleymani, S. Shateyi, P. Assari, F. K. Haghani, New mono- and biaccelerator iterative methods with memory for nonlinear equations, Abstract and Applied Analysis 705674 (2014) 1-8. 


\begin{tabular}{|c|c|c|c|c|c|c|}
\hline Test & $x_{0}$ & Method & $\left|x_{k}-x_{k-1}\right|$ & $\left|f\left(x_{k}\right)\right|$ & iter & $\mathrm{ACOC}$ \\
\hline \multirow{11}{*}{$f_{1}$} & \multirow{11}{*}{1} & NEW & $1.87 \mathrm{e}-273$ & $5.2 \mathrm{e}-546$ & 11 & 2 \\
\hline & & OST & $7.32 \mathrm{e}-139$ & $1.31 \mathrm{e}-553$ & 6 & 4 \\
\hline & & JAR & $4.09 \mathrm{e}-139$ & $1.27 \mathrm{e}-554$ & 6 & 4 \\
\hline & & PDP1 & $4.10 \mathrm{e}-237$ & $8.76 \mathrm{e}-1053$ & 6 & 4.4462 \\
\hline & & PDP2 & $7.04 \mathrm{e}-246$ & $8.50 \mathrm{e}-1092$ & 6 & 4.4443 \\
\hline & & PDP3 & $5.24 \mathrm{e}-231$ & $1.69 \mathrm{e}-1025$ & 6 & 4.4449 \\
\hline & & PDP4 & $3.56 \mathrm{e}-213$ & $3.82 \mathrm{e}-946$ & 6 & 4.4463 \\
\hline & & $\overline{\mathrm{CCT} 1}$ & $6.49 \mathrm{e}-230$ & $9.74 \mathrm{e}-1021$ & 6 & 4.4482 \\
\hline & & CCT2 & $8.62 \mathrm{e}-242$ & $1.33 \mathrm{e}-1073$ & 6 & 4.4452 \\
\hline & & CCT3 & $2.74 \mathrm{e}-162$ & $5.67 \mathrm{e}-720$ & 7 & 4.4132 \\
\hline & & CCT4 & $8.87 \mathrm{e}-121$ & $5.48 \mathrm{e}-535$ & 9 & 4.5000 \\
\hline \multirow{11}{*}{$f_{2}$} & \multirow{11}{*}{1} & NEW & $1.87 \mathrm{e}-380$ & $1.23 \mathrm{e}-760$ & 10 & 2 \\
\hline & & OST & $1.76 \mathrm{e}-258$ & $1.62 \mathrm{e}-1033$ & 6 & 4 \\
\hline & & JAR & $3.47 \mathrm{e}-286$ & $3.36 \mathrm{e}-1144$ & 6 & 4 \\
\hline & & PDP1 & $4.59 \mathrm{e}-477$ & $\overline{0}$ & 6 & 4.4520 \\
\hline & & PDP2 & $5.25 \mathrm{e}-477$ & $2.7 \mathrm{e}-2008$ & 6 & 4.4520 \\
\hline & & PDP3 & $5.56 \mathrm{e}-480$ & 0 & 6 & 4.4522 \\
\hline & & PDP4 & $4.97 \mathrm{e}-480$ & $5.39 \mathrm{e}-2008$ & 6 & 4.4522 \\
\hline & & CCT1 & $3.99 \mathrm{e}-477$ & $\overline{0}$ & 6 & 4.4519 \\
\hline & & CCT2 & $4.91 \mathrm{e}-477$ & $5.39 \mathrm{e}-2008$ & 6 & 4.4520 \\
\hline & & CCT3 & $2.78 \mathrm{e}-481$ & 0 & 6 & 4.4462 \\
\hline & & CCT4 & $7.26 \mathrm{e}-424$ & $5.27 \mathrm{e}-1886$ & 6 & 4.4490 \\
\hline \multirow{11}{*}{$f_{3}$} & \multirow{11}{*}{1} & NEW & $1.12 \mathrm{e}-333$ & $4.62 \mathrm{e}-667$ & 10 & 2 \\
\hline & & OST & $4.21 \mathrm{e}-296$ & $1.35 \mathrm{e}-1183$ & 6 & 4 \\
\hline & & JAR & $1.63 \mathrm{e}-299$ & $2.79 \mathrm{e}-1197$ & 6 & 4 \\
\hline & & PDP1 & $8.18 \mathrm{e}-126$ & $7.18 \mathrm{e}-559$ & 5 & 4.4839 \\
\hline & & PDP2 & $5.23 \mathrm{e}-126$ & $9.82 \mathrm{e}-560$ & 5 & 4.4837 \\
\hline & & PDP3 & $6.12 \mathrm{e}-123$ & $5.20 \mathrm{e}-546$ & 5 & 4.4831 \\
\hline & & PDP4 & $1.16 \mathrm{e}-123$ & $3.24 \mathrm{e}-549$ & 5 & 4.4841 \\
\hline & & CCT1 & $1.27 \mathrm{e}-125$ & $5.00 \mathrm{e}-558$ & 5 & 4.4841 \\
\hline & & CCT2 & $6.56 \mathrm{e}-126$ & $2.68 \mathrm{e}-559$ & 5 & 4.4838 \\
\hline & & CCT3 & $9.88 \mathrm{e}-458$ & $1.35 \mathrm{e}-2008$ & 6 & 4.4417 \\
\hline & & CCT4 & $4.39 \mathrm{e}-449$ & $1.87 \mathrm{e}-1997$ & 6 & 4.4414 \\
\hline
\end{tabular}

Table 3: Numerical comparison for $f_{1}, f_{2}$ and $f_{3}$.

[11] T. Lotfi, P. Assari, New three- and four-parametric iterative with memory methods with efficiency index near 2, Applied Mathematics and Computation 270 (2015) 1004-1010.

[12] T. Lotfi, F. Soleymani, P. Assari, On the construction of some tri-parametric iterative methods with-memory, Numerical Algorithms 70 (2015) 835-845.

[13] J. R. Sharma, P. Gupta, On some highly efficient derivative free methods with and without memory for solving nonlinear equations, International Journal of Computational Methods 12 (2015) 1-28.

[14] F. Soleymani, T. Lotfi, E. Tavakoli, F. K. Haghani, Several iterative methods with memory using selfaccelerators, Applied Mathematics and Computation 254 (2015) 452-458.

[15] F. Zafar, N. Yasmin, M. A. Kutbi, M. Zeshan, Construction of tri-parametric derivative free fourth order with and without memory iterative method, Journal of Nonlinear Science and Applications 9 (2016) 1410-1423.

[16] J. M. Ortega, W. G. Rheinboldt, Iterative solutions of nonlinear equations in several variables, SIAM, 1970. 


\begin{tabular}{|c|c|c|c|c|c|c|}
\hline Test & $x_{0}$ & Method & $\left|x_{k}-x_{k-1}\right| \mid$ & $\left|f\left(x_{k}\right)\right|$ & iter & $\mathrm{ACOC}$ \\
\hline \multirow{11}{*}{$f_{4}$} & \multirow{11}{*}{2.5} & NEW & $6.24 \mathrm{e}-447$ & $1.17 \mathrm{e}-892$ & 12 & 2 \\
\hline & & OST & $1.99 \mathrm{e}-490$ & $3.13 \mathrm{e}-1959$ & 7 & 4 \\
\hline & & JAR & $1.99 \mathrm{e}-490$ & $3.13 \mathrm{e}-1959$ & 7 & 4 \\
\hline & & PDP1 & $4.44 \mathrm{e}-159$ & $1.07 \mathrm{e}-704$ & 6 & 4.4468 \\
\hline & & PDP2 & $3.73 \mathrm{e}-165$ & $1.01 \mathrm{e}-731$ & 6 & 4.4480 \\
\hline & & PDP3 & $8.06 \mathrm{e}-146$ & $1.48 \mathrm{e}-645$ & 6 & 4.4470 \\
\hline & & PDP4 & $1.08 \mathrm{e}-148$ & $2.38 \mathrm{e}-658$ & 6 & 4.4468 \\
\hline & & CCT1 & $2.48 \mathrm{e}-154$ & $1.42 \mathrm{e}-683$ & 6 & 4.4458 \\
\hline & & CCT2 & $1.10 \mathrm{e}-161$ & $2.76 \mathrm{e}-716$ & 6 & 4.4474 \\
\hline & & CCT3 & $4.56 \mathrm{e}-130$ & $1.63 \mathrm{e}-575$ & 8 & 4.4758 \\
\hline & & CCT4 & $3.69 \mathrm{e}-130$ & $1.86 \mathrm{e}-576$ & 33 & 4.5065 \\
\hline \multirow{11}{*}{$f_{5}$} & \multirow{11}{*}{2} & NEW & $4.53 \mathrm{e}-288$ & $1.33 \mathrm{e}-574$ & 10 & 2 \\
\hline & & OST & $1.09 \mathrm{e}-303$ & $1.32 \mathrm{e}-1212$ & 6 & 4 \\
\hline & & JAR & $1.09 \mathrm{e}-303$ & $1.32 \mathrm{e}-1212$ & 6 & 4 \\
\hline & & PDP1 & $4.35 \mathrm{e}-448$ & $4.03 \mathrm{e}-1991$ & 6 & 4.4485 \\
\hline & & PDP2 & $3.58 \mathrm{e}-455$ & $2.16 \mathrm{e}-2007$ & 6 & 4.4480 \\
\hline & & PDP3 & $1.38 \mathrm{e}-430$ & $4.13 \mathrm{e}-1913$ & 6 & 4.4485 \\
\hline & & PDP4 & $1.23 \mathrm{e}-427$ & $5.56 \mathrm{e}-1900$ & 6 & 4.4486 \\
\hline & & CCT1 & $3.48 \mathrm{e}-442$ & $7.52 \mathrm{e}-1965$ & 6 & 4.4490 \\
\hline & & CCT2 & $1.57 \mathrm{e}-451$ & $1.94 \mathrm{e}-2006$ & 6 & 4.4483 \\
\hline & & CCT3 & $8.38 \mathrm{e}-264$ & $3.60 \mathrm{e}-1171$ & 6 & 4.4440 \\
\hline & & CCT4 & $1.66 \mathrm{e}-247$ & $1.18 \mathrm{e}-1098$ & 6 & 4.4442 \\
\hline \multirow{11}{*}{$f_{6}$} & \multirow{11}{*}{1} & NEW & $2.27 \mathrm{e}-303$ & $9.77 \mathrm{e}-606$ & 11 & 2 \\
\hline & & OST & $3.94 \mathrm{e}-187$ & $8.34 \mathrm{e}-747$ & 6 & 4 \\
\hline & & JAR & $3.61 \mathrm{e}-184$ & $6.54 \mathrm{e}-735$ & 6 & 4 \\
\hline & & PDP1 & $1.77 \mathrm{e}-214$ & $1.02 \mathrm{e}-951$ & 6 & 4.4489 \\
\hline & & PDP2 & $1.83 \mathrm{e}-224$ & $3.81 \mathrm{e}-996$ & 6 & 4.4493 \\
\hline & & PDP3 & $1.65 \mathrm{e}-197$ & $4.89 \mathrm{e}-876$ & 6 & 4.4492 \\
\hline & & PDP4 & $3.66 \mathrm{e}-200$ & $7.55 \mathrm{e}-888$ & 6 & 4.4490 \\
\hline & & CCT1 & $3.05 \mathrm{e}-207$ & $1.61 \mathrm{e}-919$ & 6 & 4.4483 \\
\hline & & CCT2 & $1.27 \mathrm{e}-218$ & $3.76 \mathrm{e}-970$ & 6 & 4.4491 \\
\hline & & CCT3 & $1.24 \mathrm{e}-463$ & $1.35 \mathrm{e}-2008$ & 8 & 4.4475 \\
\hline & & CCT4 & $1.70 \mathrm{e}-346$ & $3.13 \mathrm{e}-1539$ & 9 & 4.4374 \\
\hline
\end{tabular}

Table 4: Numerical comparison for $f_{4}, f_{5}$ and $f_{6}$.

[17] H. T. Kung, J. F. Traub, Optimal order of one-point and multipoint iteration, Journal of the Association for Computing Machinery 21 (4) (1974) 643-651.

[18] A. M. Ostrowski, Solutions of equations and systems of equations, Academic Press, 1966.

[19] B. Campos, A. Cordero, J. R. Torregrosa, P. Vindel, A multidimensional dynamical approach to iterative methods with memory, Applied Mathematics and Computation 271 (2015) 701-715.

[20] B. Campos, A. Cordero, J. R. Torregrosa, P. Vindel, Stability of King's family of iterative methods with memory, Journal of Computational and Applied Mathematics 318 (2017) 504-514.

[21] R. C. Robinson, An introduction to dynamical systems: continuous and discrete, American Mathematical Society, 2012.

[22] F. I. Chicharro, A. Cordero, J. R. Torregrosa, Drawing dynamical and parameters planes of iterative families and methods, The Scientific World Journal 2013 (780513) (2013) 1-11. 


\begin{tabular}{|c|c|c|c|c|c|c|}
\hline Test & $x_{0}$ & Method & ||$x_{k}-x_{k-1} \|$ & $\left|f\left(x_{k}\right)\right|$ & iter & ACOC \\
\hline \multirow{5}{*}{$f_{7}$} & & NEW & $7.79 \mathrm{e}-352$ & $4.91 \mathrm{e}-702$ & 11 & 2 \\
& & OST & $1.48 \mathrm{e}-187$ & $7.06 \mathrm{e}-748$ & 6 & 4 \\
& & JAR & $1.48 \mathrm{e}-187$ & $7.06 \mathrm{e}-748$ & 6 & 4 \\
\cline { 3 - 7 } & & PDP1 & $3.03 \mathrm{e}-300$ & $3.9 \mathrm{e}-1333$ & 6 & 4.4467 \\
& & PDP2 & $1.07 \mathrm{e}-307$ & $2.66 \mathrm{e}-1366$ & 6 & 4.4456 \\
& & PDP3 & $1.66 \mathrm{e}-288$ & $8.99 \mathrm{e}-1281$ & 6 & 4.4461 \\
& & PDP4 & $3.30 \mathrm{e}-277$ & $1.70 \mathrm{e}-1230$ & 6 & 4.4468 \\
\cline { 3 - 7 } & & CCT1 & $4.16 \mathrm{e}-294$ & $8.12 \mathrm{e}-1306$ & 6 & 4.4480 \\
& & CCT2 & $4.97 \mathrm{e}-304$ & $5.56 \mathrm{e}-1350$ & 6 & 4.4461 \\
& & CCT3 & $9.06 \mathrm{e}-444$ & $8.92 \mathrm{e}-1972$ & 8 & 4.4446 \\
& & CCT4 & $8.53 \mathrm{e}-348$ & $9.18 \mathrm{e}-1545$ & 7 & 4.4406 \\
\hline \multirow{5}{*}{$f_{8}$} & NEW & $3.29 \mathrm{e}-289$ & $2.37 \mathrm{e}-866$ & 8 & 3 \\
& & OST & $4.16 \mathrm{e}-302$ & $2.75 \mathrm{e}-1508$ & 6 & 5 \\
& & JAR & $1.61 \mathrm{e}-361$ & $1.78 \mathrm{e}-1605$ & 6 & 5 \\
\cline { 3 - 8 } & & PDP1 & $2.15 \mathrm{e}-119$ & $2.94 \mathrm{e}-678$ & 5 & 5.7371 \\
& & PDP2 & $2.28 \mathrm{e}-119$ & $4.10 \mathrm{e}-678$ & 5 & 5.7371 \\
& & PDP3 & $2.31 \mathrm{e}-119$ & $4.38 \mathrm{e}-678$ & 5 & 5.7371 \\
& & PDP4 & $2.31 \mathrm{e}-119$ & $4.35 \mathrm{e}-678$ & 5 & 5.7371 \\
\cline { 3 - 7 } & & CCT1 & $2.03 \mathrm{e}-119$ & $2.11 \mathrm{e}-678$ & 5 & 5.7372 \\
& & CCT2 & $2.22 \mathrm{e}-119$ & $3.47 \mathrm{e}-678$ & 5 & 5.7371 \\
& & CCT3 & $6.88 \mathrm{e}-180$ & $1.85 \mathrm{e}-1022$ & 5 & 5.8234 \\
& & CCT4 & $3.77 \mathrm{e}-108$ & $2.75 \mathrm{e}-614$ & 5 & 5.7108 \\
\hline
\end{tabular}

Table 5: Numerical comparison for $f_{7}$ and $f_{8}$.

[23] J. L. Varona, Graphic and numerical comparison between iterative methods, The Mathematical Intelligencer 24 (2002) 37-46.

[24] Á. A. Magreñán, A new tool to study real dynamics: The convergence plane, Applied Mathematics and Computation 248 (2014) 215-224.

[25] S. Amat, S. Busquier, S. Plaza, Chaotic dynamics of a third-order Newton-type method, Journal of Mathematical Analysis and Applications 366 (2010) 24-32.

[26] A. Cordero, J. R. Torregrosa, Variants of Newton's method using fifth-order quadrature formulas, Applied Mathematics and Computation 190 (2007) 686-698. 